\title{
0 efeito da temperatura sobre os equilíbrios de oxigênio das hemoglobinas de peixe em relação à variabilidade tér mica ambiental. (*)
}

Dennis A. Powers ('); Joseph P. Martin ( $\left.{ }^{2}\right)$; Robert L. Garlick ( ${ }^{(3)}$; Hans Jorgen Fyhn ( ${ }^{4}$ ) ; Unni E. H. Fyhn (

\section{Resumo}

Foram medidas as dependências da temperatura do equilibrio de oxigênio do sangue e das soluçōes de hemoglobina de quatro peixes neotropicais e três da zona temperada. Diferenças significativas existem nas temperaturas de oxigenaçẫo dos sangues, porém não das solu. ções de hemoglobina de diferentes espécies. Diferenças na sensibilidade térmica de oxigenação dos sangues parecem depender das diferenças no $\mathrm{pH}$ intracelular, nos fosfatos orgânicos e nas outras variávels da uniâo de ligantes e das entalpias intrínsecas da oxigenaçāo das hemoglobinas em si.

\section{INTRODUÇÃo}

Bioquímicos e fisiologistas comparativos tem fornecido valiosa informaçâo com respeito à maquinária molecular para adaptar-se à tensão ambiental (Prosser, 1973; Smellie \& Pennock, 1976). Como os poiquilotermas devem adaptar-se às mudanças térmicas, foram feitos esforços para estudar seus mecanismos adaptativos (ver as revisões de Prosser, 1967; Hazel \& Prosser, 1974) .

As necessidades de oxigênio dos peixes estão correlacionadas com a temperatura. Por exemplo, Fry \& Hart (1948) mostraram que o oxigênio consumido pelas carpas douradas é aumentado em cerca de $250 \%$ sobre uma variação de $10^{\circ} \mathrm{C}$. Conforme a temperatura aumenta, a disponibilidade de oxigênio na água diminui porque: (1) a solubilidade de oxigênio diminui com o aumento da temperatura (Lei de Henry), e (2) a elevada atividade biológica (e.g., bactérica e planctônica) a altas temperaturas muitas vezes reduzem o oxigênio à baixa saturaçōes. Conseqüentemente, em temperaturas mais altas, os peixes necessitam de mais oxigênio porém é menos disponivel em seu meio ambiente.
Em geral, os peixes respondem a este dilema por uma combinação de estratégias. Por exemplo, o volume de ventilação e a batida do coração aumentam diretamente com a temperatura (Prosser, 1973). Alguns peixes aumentam a capacidade de transporte do oxigênio do sangue pelo aumento do hematócrito (DeWilde \& Houston, 1967; Cameron, 1970; Powers, 1974; Houston \& Cry, 1974; Powers \& Powers, 1975). Entretanto, a evidência recente sugere que primariamente este fenômeno é induzido estacionalmente ao invés de uma resposta à temperatura per se (Denton \& Yousef, 1975; Greaney \& Powers, 1977a). As vezes, há mudanças nos niveis intracelulares dos modificadores alostéricos (e.g., Greaney \& Powers, 1977b) que, em troca, aumentam a afinidade pelo oxigênio da hemoglobina às temperaturas mais elevadas.

O efeito da temperatura sobre os equilíbrios de oxigênio da hemoglobina dos peixes foi revisado (Riggs, 1970; Johansen \& Lenfant, 1972; Johansen \& Weber, 1976). Embora o número de espécies examinadas seja limitado, as propriedades funcionais das hemoglobinas dos teleósteos podem ser divididas em três categorias maiores como sugeridas por Weber et al., (1976). A classe I contém espécies com uma ou mais hemoglobinas, todas sensíveis tanto à temperatura como ao $\mathrm{pH}$ (e.g., Gillen \& Riggs, 1971; Gillen \& Riggs, 1972; Bonaventura, Gillen \& Riggs, 1974; Weber, 1975; Mied \& Powers, 1977). A classe II tem espécies com componentes múltiplos, alguns dos quais são funcionalmente semelhantes às hemoglobinas, da classe I, enquanto os outros componentes não são atingidos tanto pela temperatura como pelo $\mathrm{pH}$ (e..g. Hashimoto et al.,

(•) - Versāo original inglesa publicada em Comp. Biochem. Physiol. vol. 62A (1). 1979.

(1) - Department of Biology, The Johns Hopkins Univer sity, Baltimore, Maryland 21218, U.S.A.

( 2 ) - Duke University Marine Laboratory, Beaufort, North Carolina 28516, U.S.A.

(3) - Departament of Zoology, University of Texas, Austin, Texas 78712, U.S.A.

(4) - Institute of Zoophysiology, University of Oslo, Blindern, Oslo 2, Norway. 
1960; Yamaguchi et al., 1962; Yamaguchi et al., 1963; Binotti et al., 1971; Powers \& Edmundson, 1972; Powers, 1972; Powers, 1974; Wyman et al., 1977). Os peixes da classe III possuem hemoglobinas que são sensíveis ao pH mas são insensíveis à temperatura (e.g. . Rossi-Fanelli \& Antonini, 1960; Anderson et. al., 1973). Estes estudos mostraram que entre as hemoglobinas do peixe, a entalpia aparente $(\Delta H)$ da oxigenação varia de zero a cerca de 16 kcai por mol. Johansen \& L'enfant (1972) asseveram: "parece bem estabelecido que o desenvolvimento evolucionário favorece uma diminuição na sensibilidade da temperatura da afinidade de $\mathrm{O}_{2}-\mathrm{Hb}$ nas espécies que experimentam grandes flutuações na temperatura".

Nosso estudo, analisa-se a afinidade pelo oxigênio das hemoglobinas de quatro peixes amazônicos e comparam-se os resultados com os dados de equilíbrio de outras espécies de peixe. Em complemento, os estudos de equilíbrio de oxigênio do sangue são discutidos para diversas espécies em relação aos dados obtidos com as soluções de hemoglobina.

\section{MATERIAL \& MÉTODOS}

Os organismos empregados neste estudo foram colhidos e sangrados segundo o método esboçado por Fyhn et al. (1978).

As curvas de oxigenação do sangue total foram determinadas com um analisador de dis. sociação de oxigênio Aminco Hem-O-Scan conforme descrito por Powers et al. (1978). Os estudos de equilibrio de oxigênio dos componentes da hemoglobina foram realizados pelo micro método esboçado abaixo. Os componentes individuais e as amostras totais de hemoglobina foram separados do fosfato orgânico e de outros íons pela passagem das soluções de hemoglobina primeiro por uma coluna G-25 Sephadex $(110 \times 1,5 \mathrm{~cm})$ equilibrada com Tris$\mathrm{HCl} 1 \mathrm{mM}, \mathrm{pH} 8,2$, e depois por uma coluna separadora de troca de íons segundo o método de Dintzis (1952). As hemoglobinas purificadas foram ou diluídas diretamente com tampão ou dializadas contra o Tris a $0,1 \mathrm{M}, \mathrm{pH} 9,0$ ou o tampão"de borato $0,15 \mathrm{M}$, pH 9,0 de cada solução de hemoglobina foi então medido com um micro elétrodo de $\mathrm{pH}$ do Radiômetro com correções apropriadas para a temperatura. As concentraçōes de hemoglobina estiveram entre $0,1 \mathrm{mM}$ e $4 \mathrm{mM}$. As concentrações de hemoglobina humana foram de $2,7 \mathrm{mM}$. Uma aliquota de 3 ul de amostra foi colocada sobre uma lâmina de vidro de tampa redonda (espes. sura número 2 de $18 \mathrm{~mm}$ ) e coberta por uma peça circular de $18 \mathrm{~mm}$ de uma membrana de um copolimero silicone (General Eletric Co.). A amostra foi tampada com o dispositivo de montagem de amostras "Aminco" e colocada no portador de amostras do analisador de dissociação de oxigênio de "Aminco Hem-O-Scan" até que a amostra ficasse equilibrada à temperatura e às pressões parciais dos gases na máquina. $\mathrm{O}$ analisador foi calibrado de acordo com a pressão barométrica atual. A amostra foi depois desoxigenada com um nitrogênio de pureza ultra-elevada $(99,999 \%)$ e o nível de oxigenação numa porcentagem de zero foi estabelecida. A oxigenação foi depois ajustada à calibragem mais baixa $(5 \mathrm{~mm} \mathrm{~g} / \mathrm{min})$. A expansão do eixo-X (pressão parcial do oxigênio) foi acompanhada por uma série de resistências calibradas. Mais pormenores do método são dados por Powers et al. (1978) .

\section{Resultados}

A energia livre total da oxigenação, $\Delta \mathrm{G}$, é a soma das energias livres de oxigenação para cada uma dos quatro passos. A energia livre total pode ser calculada por :

$$
\Delta \mathrm{G}=-\mathrm{RT} \ln \mathrm{K}
$$

onde $\mathrm{R}$ é a constante universal de gás, $\mathrm{T}$ é a temperatura absoluta e K é a constante termodinâmica de equilibrio. Wyman (1964) mostrou que a equação acima poderia ser definida em termos da atividade média do ligante $X_{m}$ por :

$$
\Delta \mathrm{G}=+\mathrm{RT} \ln \mathrm{X}_{\mathrm{m}}
$$

a qual é a mudança de energia livre quando saturando hemoglobina com ligante $X$. Em nosso estudo, $X$ é o oxigênio e $\Delta G$ é expresso em quilocalorias por mol de ligante. Como a concentração média de oxigênio foi aproximadamente igual à concentração de oxigênio em que a hemoglobina foi saturada na metade $\left(P_{s_{0}}\right)$, usamos o $P_{50}$, para nossos cálculos. 
A entalpia de van't Hoff, $\Delta H$, pode ser calculada da inclinação do ploteado de van't Hoff segundo a equaçäo:

$$
\Delta H=2,303 R \quad \frac{d \log P_{50}}{d(1 / T)}
$$

As figuras de 1 a 7 são os plotados de van't $\mathrm{H}_{\mathrm{off}}$ mostrando os dados da uniāo de $\mathrm{O}_{2}$ para o sangue e hemoglobinas das espécies representativas dos peixes de diferentes ambientes térmicos. A figura 1 mostra os dados para - Fundulus heteroclitus, uma carpa eurialina, encontrada em águas temperadas ao longo da costa leste dos Estados Unidos e Canadá. Esta figura demonstra claramente que os glóbulos vermelhos de Fundulus na presença de $\mathrm{CO}_{2}$ (i.e.. pH mais baixo) são menos afetados pelas mudanças na temperatura do que os eritrócritos equilibrados com gases sem $\mathrm{CO}_{2}$. Além do mais, ambos os experimentos dos glóbulos vermelhos ilustram a sensibilidade térmica reduzida do sangue em comparação às soluçōes de hemoglobina em pH alcalino.

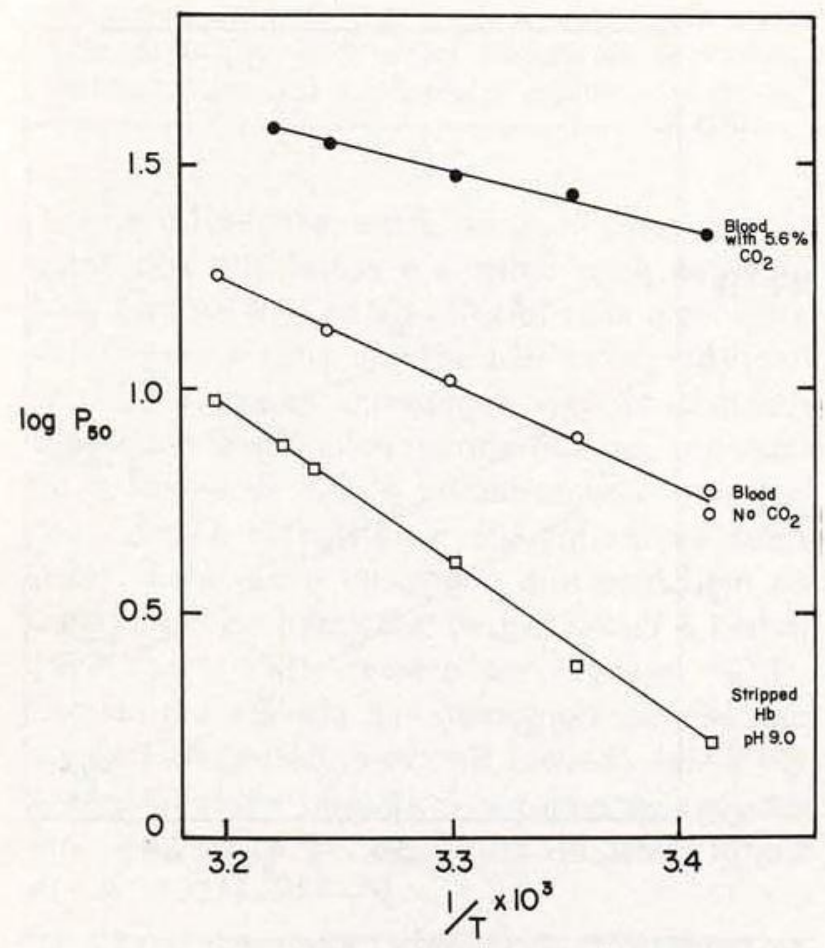

Fig. 1 - $O$ efeito da temperatura sobre a afinidade de oxigênlo de Fundulus heteroclitus : (a) sangue na presença de $5,6 \%$ de $\mathrm{CO}_{2}(--)$; (b) sangue na ausêncla de $\mathrm{CO}_{2}(-0-)$; e solução de hemoglobina $(\mathrm{pH} 9,0)$ fraclonada dos fosfatos orgânicos $(-\square-)$.
A fig. 2 é um plotado de van't $\mathrm{H}_{\mathrm{Off}_{\mathrm{ff}}}$ do sangue e hemoglobina da arraia (Raia sp), que é um residente não passageiro perto de

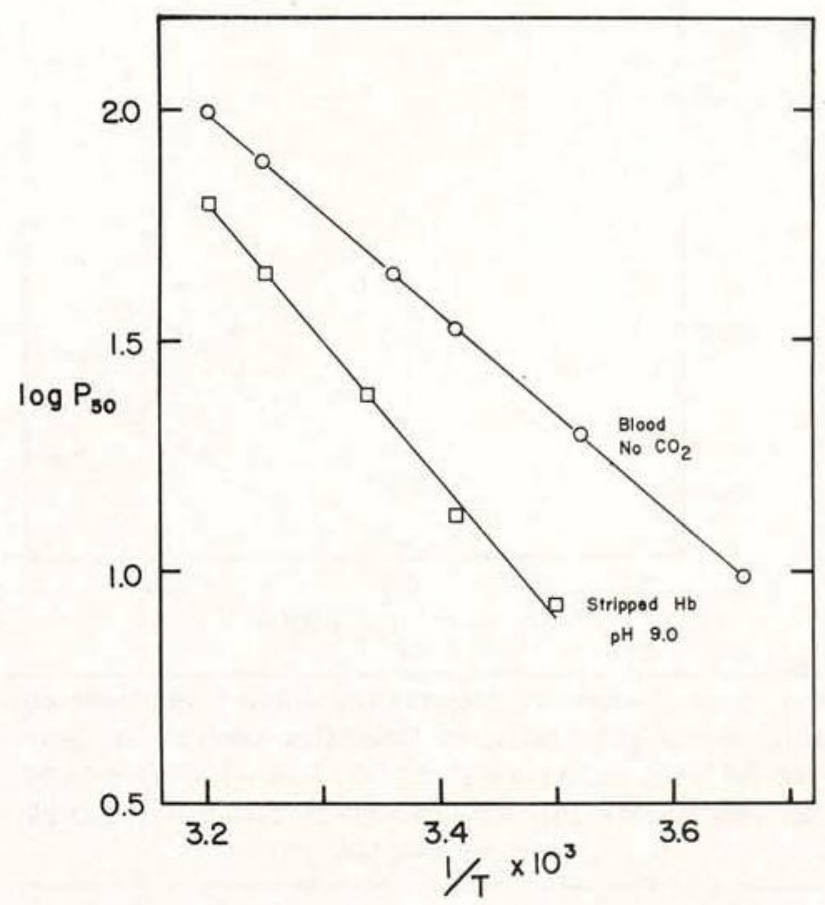

Fig. 2 - 0 efeito da temperatura sobre a afinidade de oxigênio da arraia (Raia sp.): (a) sangue na ausência de $\mathrm{Co}_{2}(-0)$; e (b) solução de hemoglobina $(\mathrm{pH} 9,0)$ fracionada dos fosfatos orgânicos $(-\square-)$.

Woods Hole, Mass., e a Fig. 3 é um plotado de van't $\mathrm{H}_{\mathrm{off}_{\mathrm{ff}}}$ de solução de hemoglobina e do sangue do peixe-cachorro (Mustelus canis), apanhado perto de Woods Hole. Estes dois pelxes cartilaginosos são encontrados em embientes com grandes flutuações térmicas anuais (Anônimo, 1968). Evidentemente, ambos os peixes têm sangue e hemoglobina que são afetados pela temperatura e as inclinações destes plotados de van't $\mathrm{H}_{\mathrm{ff}}$ são iguais ou maiores do que as de outras espécies de meios ambientes com pouca ou nenhuma flutuação térmica anual (ver Figs. 4-6). As figuras 4-6 representam os dados para três peixes amazônicos (Lepidosiren paradoxa, Serrasalmus rhombius e Arapaima gigas). Estas espécies mostraram um efeito menor de temperatura nos glóbulos vermelhos do que na hemoglobina fracionada, em $\mathrm{pH} 9,0$. Além disso, quando o $\mathrm{CO}_{2}$ esteve presente nos gases de equilíbrio, as glóbulos 


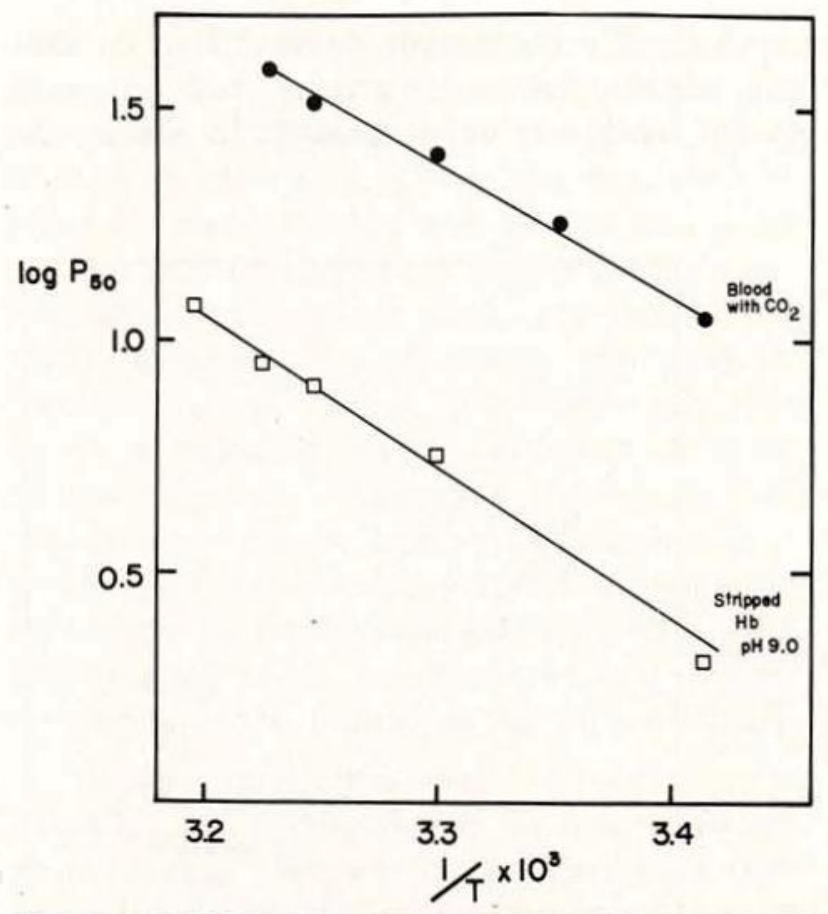

Fig. 3-O efeito da temperatura sobre a afinidade de oxigênio do peixe-cachorro (Mustelus canis): (a) sangue na presença de $5,6 \%$ de $\mathrm{CO}_{2}(-\bullet-)$; (b) soluçăo de hemoglobina $(\mathrm{pH} 9,0)$ fracionada dos fosfatos orgânicos (- - -).

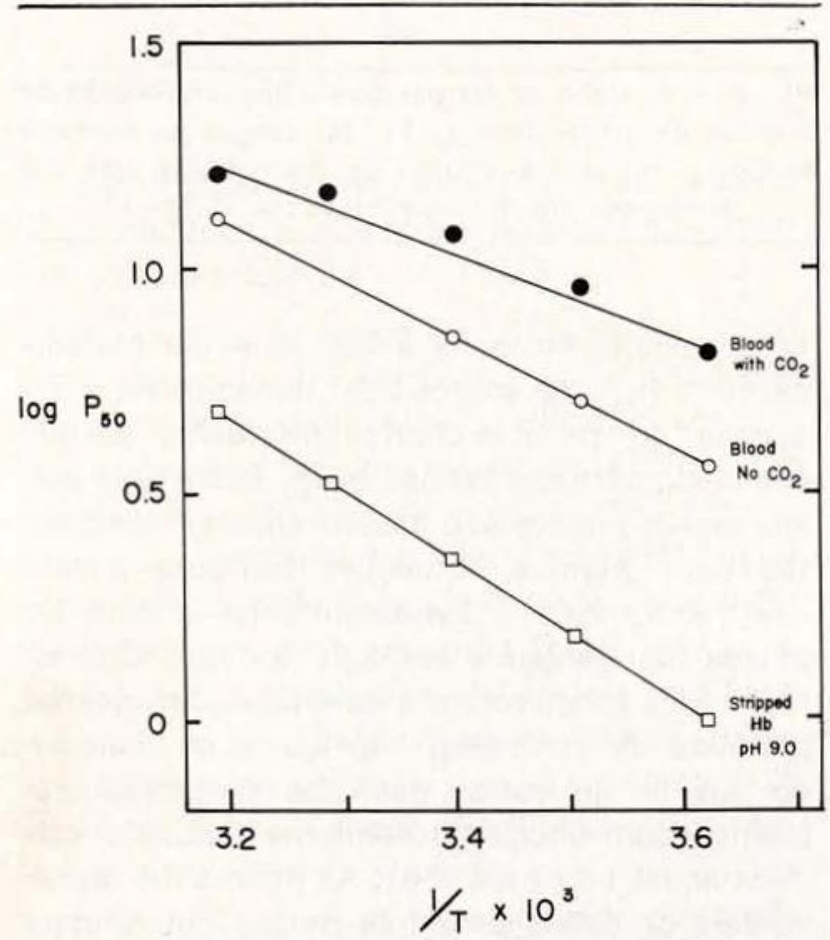

Fig. 4- $\mathrm{O}$ eteito da temperatura sobre a afinidade de oxigênio do peixe pulmonado (Lepidosiren paradoxa): (a) sangue na presença de $5,6 \%$ de $\mathrm{CO}_{2}(-\bullet-)$; (b) sangue na mausência de $\mathrm{CO}_{2}(-0-)$; e (c) solução de hemoglobina $(\mathrm{pH} 9,0)$ fracionada dos fosfatos orgânicos $(-\square-)$. vermelhos demonstraram menos sensibilidade térmica que os glóbulos equilibrados com uma atmosfera que não continha $\mathrm{CO}_{2}$. De interesse adicional é um desvio aparente da linearidade nos dados do sangue exposto ao $\mathrm{CO}_{2}$. Como estes desvios foram sempre maiores em temperaturas mais baixas, os dados sugerem um ou mais das seguintes explorações: (1) inibição térmica do transporte de $\mathrm{CO}_{2}$ nos glóbulos vermelhos, (2) uma função catalítica reduzida de anidrase carbônica nestas espécies de água temperada de meio ambiente constante, (3) variação do $\mathrm{pH}$ do sangue entre indivíduos, (4) mudança no $\mathrm{pH}$ do sangue como uma função de temperatura ou (5) desnaturação pelo calor a temperaturas mais elevadas. Geralmente, as Figuras de 1 a 6 ilustram que todas as seis espécies de peixes demonstram respostas térmicas semelhantes. Isto é, a hemoglobina fracionada $(\mathrm{pH} 9,0)$ é a mais sensível à temperatura, os glóbulos vermelhos sem $\mathrm{CO}_{2}$ são menos sensíveis do que as hemoglobinas, e os glóbulos vermelhos são os menos sensíveis termicamente, na presença de $\mathrm{CO}_{2}$.

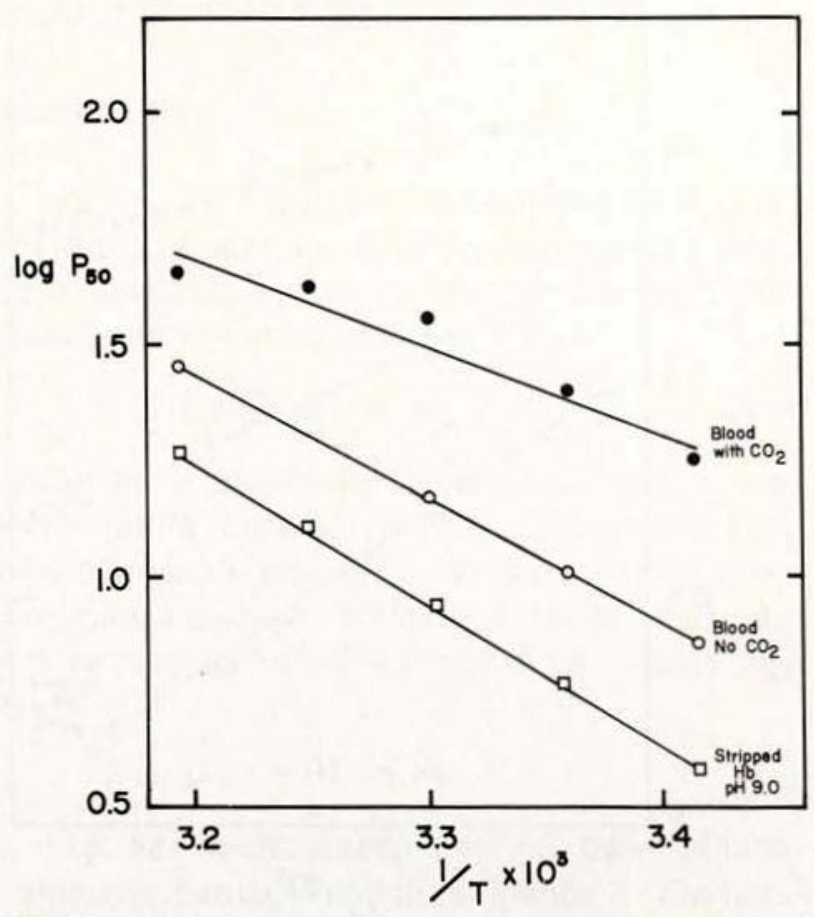

Fig. 5 - $\mathrm{O}$ efeito da temperatura sobre a afinidade de oxigênio da piranha-vermelha (Serrasalmus rhombius) : (a) sangue na presença de $5,6 \%$ de $\mathrm{CO}_{2}(--)$; (b) sangue na ausência de $\mathrm{CO}_{2}(-0-)$; e (c) solução de hemoglobina $(\mathrm{pH} \mathrm{9.0)} \mathrm{rfacionada} \mathrm{dos} \mathrm{fosfatos} \mathrm{orgânicos}$ (-口-). 


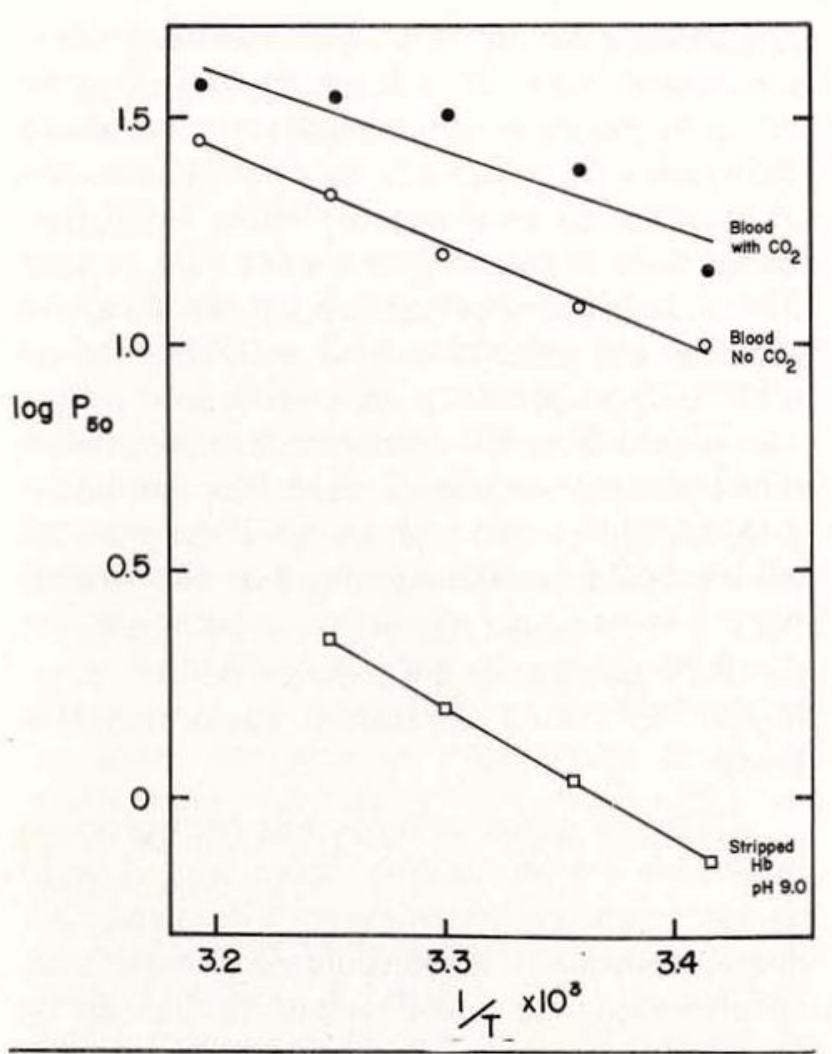

Fig. 6-O efeito da temperatura sobre a afinidade de oxigênio do pirarucu (Arapaima gigas): (a) sangue na presença de $5,6 \%$ de $\mathrm{CO}_{2}(-\bullet-) ;$ (b) sangue na ausência de $\mathrm{CO}_{2}(-0-)$; e (c) soluçăo de hemoglobina (pH 9,0) fracionada dos fosfatos orgânicos $(-\square-)$.

As diferenças entre os equilíbrios de oxigênio dos eritrócitos e a solução de hemoglobina $(\mathrm{em} \mathrm{pH} 9,0)$ estão relacionadas a $\mathrm{pH}$ intracelular e a outras variáveis da união de ligante. De fato, as diferenças na sensibilidade térmica dos glóbulos vermelhos na presença ou ausência de $\mathrm{CO}_{2}$ é primariamente uma função do $\mathrm{CO}_{2}$ dissolvido e sua influência sobre $\sigma \mathrm{pH}$. Este é um fenômeno que está bem documentado na literatura (e.g., Krogh \& Leitch, 1919; Root, 1931; revisto por Prosser, 1973). Enquanto a maioria das hemoglobinas são sensíveis à temperatura em $\mathrm{pH}$ elevado $(\mathrm{dH}$ 9-10) , a sensibilidade térmica é geralmente reduzida em pH baixo (e.g., veja Truta Hb IV em Binotti et al., 1971).

Como o sangue coletado de diferentes espécies tem diferentes pH's e fosfatos orgânicos que, por vez, afetam o $\mathrm{pH}$ intracelular e os equilíbrios de oxigênio da hemoglobina, pareria apropriado comparar os dados de hemoglobina coletados sob condições idênticas. A Fi- gura 7 mostra os dados de equilíbrio de oxigênio da hemoglobina em $\mathrm{pH} 9,0$ para as hemoglobinas 'de sete espécies de peixes e para a $\mathrm{HbA}$ humana e da mioglobina da baleia. Evidentemente, todas as espécies relacionadas na Fig. 7 têm hemoglobinas com calor de oxige-

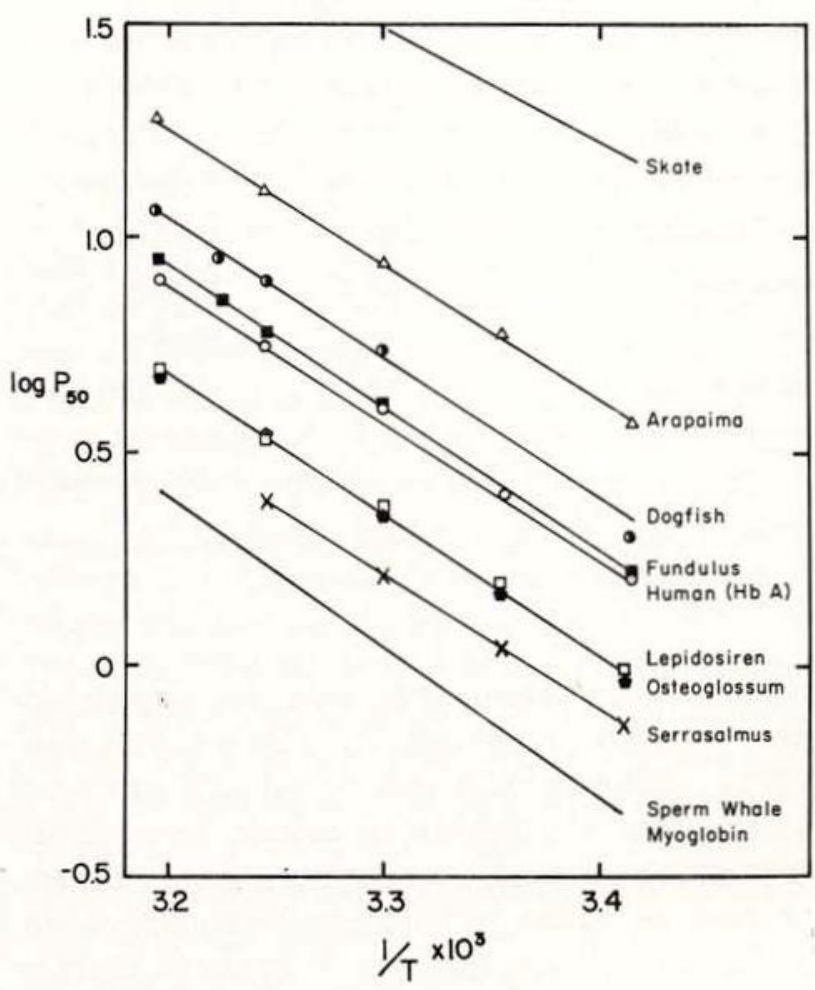

Flg. 7 - Os ploteados de van't $\mathrm{H}$ off para as soluçöes de hemoglobina $(\mathrm{pH} 9,0)$ de um número de espécies. As espécies são identificadas na figura. Um ploteado para a mioglobina de cachalote de baleia $(\mathrm{pH} 7,0)$ de Amiconi, G.. Antonini, E. Brunori, M. e Magnusson, E., cotada por Antonini \& Brunori (1971).

nação similares ( $\Delta$ H's entre $-13,7$ e $-15,8$ $\mathrm{Kcal} / \mathrm{mole}$ ) sob condiçöes essencialmente idênticas. Os valores de $\Delta \mathrm{H}$ calculados das inclinações dos plotados de van't $\mathrm{H}_{\mathrm{fff}}$ estão presentes na Tabela 1.

As afinidades de oxigênio das soluções de hemoglobina a $20^{\circ} \mathrm{C}$ (sem $\mathrm{NaCl}$ ) para três das espécies amazônicas, mostraram $\mathrm{P}_{50}$ 's muito semelhantes aos determinados na presença de $\mathrm{NaCl} 0,1 \mathrm{M}$. Entretanto, a hemoglobina de Arapaima possuia um $\mathrm{P}_{50}\left(\mathrm{NaCl} 0,1 \mathrm{M} 20^{\circ} \mathrm{C}\right)$ que era aproximadamente $2 \mathrm{~mm} \mathrm{Hg}$ maior que o determinado na ausência de sal. Parece que a hemoglobina de Arapaima tem um efeito salino significativo mesmo em $\mathrm{pH}$ relativamente 
TABELA 1 - Valores de calores de oxigenação para as hemoglobinas de peixe em pH alcalino com HbA humana e mioglobina de baleia para comparação

\begin{tabular}{lcc}
\hline Espécles & $\begin{array}{c}\Delta \text { H } \\
\text { (Kcals mol-1) }\end{array}$ & $\begin{array}{c}\text { Meio térmico } \\
\text { interno }\end{array}$ \\
\hline Human HbA & -14.6 & Constante \\
Arapaima & -14.6 & Constante \\
Lepldosiren & -15.3 & Constante \\
Osteoglossum & -15.7 & Constante \\
Serrasalmus & -13.7 & Variável \\
Mustelus & -15.3 & Variável \\
Rala & -13.7 & Variável \\
Fundulus & -15.8 & Variável \\
\hline
\end{tabular}

Inclui o calor da soluçăo para o oxigênio (a $200 \mathrm{C}$ é cerca de $-3,1$ Kcals mol -1).

- Uma hemoglobina de arraio tem um pequeno efeito Bohr mesmo em pH 9,0 (Mumm et al.. 1978).

elevado. Um efeito parecido foi registrado para carpa $\mathrm{Hbl}$ em pH 7,7 (Gillen \& Riggs, 1972). Entretanto, é concebível que um erro sistemático não detectado causou uma estratagema no perfil da temperatura especialmente para os dados colhidos na hemoglobina de Arapaima na ausência de sal. Estamos correntemente repetindo esses estudos para exami nar essa possibilidade.

\section{DISCUSSÃo}

Barcroft \& King (1909) foram os primeiros a demonstrar que um aumento na temperatura diminuirá a afinidade da hemoglobina pelo oxigênio. A determinação da sensibilidade térmica da hemoglobina pode ser feita ou por microcalorimetria ou pelo emprego do ploteado de van't Hoff. Entretanto, os dados obtidos dos ploteados de van't $\mathrm{H}_{\text {off }}$ podem ser enganosos a menos que interpretados com respeito às condições exatas sob as quais foram feitas as mediçōes. A temperatura atinge uma ampla variedade de fenômenos bioquímicos que podem influir direta ou indiretamente nos equilibrios de oxigênio. Por exemplo, a temperatura, atinge" a fluidez das membranas dos eritrócitos, o pH do sangue, as constantes de união dos fosfatos orgânicos e os coeficientes de atividade de um número de componentes intra e exrtacelulares. A entalpia de van't $\mathrm{H}_{\mathrm{off}_{\mathrm{ff}}}$ total, $\Delta \mathrm{H}$, representa a temperatura intrínseca da ligação de oxigênio e as contribuições devidas a outros processos da uniẩo de ligante. Semelhante processo é associado com os equilíbrios de próton das cadeias laterais do amino ácido (i.e., os Efeitos Root e Bohr). Em pH alcalino (i.e. pH 9-10), os efeitos Root e Bohr são essencialmente inoperantes. Conseqüentemente, os ploteados de van't $\mathrm{H}_{\mathrm{o}_{\mathrm{ff}}}$ das hemoglobinas em pH 9,0 são em geral isentas das contribuições entálpicas devidas aos efeitos Root e Bohr como também outros processos de união de ligante dependente de $\mathrm{pH}$ (e.g. ligação do fosfato orgânico à desoxi-hemoglobina) .

Como os dados colhidos nas hemoglobinas fracionada em $\mathrm{pH}$ alcalino, sobre uma variação de temperaturas, fornecessem informação primariamente sobre a molécula da hemoglobina, as diferenças ou semelhanças de ligação de $\mathrm{O}_{2}$ observadas entre espécies deveriam refletir os parâmetros termodinâmicos intrínsicos das hemoglobinas per se. Sob tais condições as inclinações dos ploteados van't $H_{\text {off }}$ para as nemoglobinas fracionadas (e.g., veja Fig. 7) foram quase idênticas. Além disso, estes dados estão de acordo com os valores para um número de hemoglobinas de peixe de uma variedade de ambientes térmicos. Visto que a energia livre total de oxigenação $(\Delta G)$ é diferente para cada uma das várias hemoglobinas. (Como: $\Delta G=+R T$ In $X_{m}$ ) segue-se que a maior diferença termodinâmica entre espécies com entalpias idênticas ou semelhantes, $\Delta H$, deveria ser primariamente entrópica porque :

$$
\Delta \mathrm{G}=\Delta \mathrm{H}-\mathrm{T} \Delta \mathrm{S}
$$

Soluçōes diferentes de hemoglobina em $\mathrm{pH}$ alcalino, os dados de equilibrio do oxigênio do sangue apresentados tanto aqui como na literatura (e.g., Johansen e Lenfant 1972) mostraram contribuições substanciais da protonação e outros fenômenos associados. $\mathrm{Na}$ presença de $\mathrm{CO}_{2} \circ \mathrm{pH}$ do sangue será baixo, reduzindo, além disso, a sensibilidade térmica aparente das curvas de equilíbrio de oxigênio (e.g., veja Figuras 1-6). 
Johansen \& Lenfant (1972) compararam os dados para a hemoglobina humana (Benesch \& Benesch, 1970) com dados para o sangue de seis espécies (Rossi Fanelli \& Antonini, 1960; Grigg, 1967; Lenfant \& Johansen, 1968; Johansen et al., 1970; Lenfant et al., 1966 e Dill et al., 1932). Alguns destes estudơs de sangue foram feitos na ausência de $\mathrm{CO}_{2}$ enquanto outros foram feitos na presença de $\mathrm{CO}_{2}$. Comparando a afinidade de $\mathrm{O}_{2}-\mathrm{Hb}$ de diversas espécies sob uma série de diferentes condições bioquímicas e fisiológicas torna-se difícil a interpretação e avaliação das conclusões. Talvez os estudos comparativos poderiam considerar a possibilidade que um grande efeito da temperatura sobre a afinidade de $\mathrm{O}_{2}-\mathrm{Hb}$ podia ser mais propriamente interpretado como um efeito diminuido de outros parâmetros de união de ligante (e.g., o papel dos fostatos orgânicos e pH) .

Como previamente estabelecido, foi proposto (Johansen \& Lenfant, 1972), que a evolução tenha optado por uma diminuição na sensibilidade de temperatura das hemoglobinas nas espécies que experimentam grandes flutuações térmicas do meio ambiente, enquanto as espécies que vivem em meios térmicos relativamente constantes têm sensibilidades térmicas mais elevadas. Esta generalização conta com diversas linhas de evidência. Primeiro, os peixes da Antártica (e.g., Trematomous), que vivem em ambientes frios, mas anualmente constantes, apresentam grande sensibilidade térmica $(\Delta \mathrm{H}=-11 \mathrm{Kcal} / \mathrm{mol})$ da afinidade de $\mathrm{O}_{2}-\mathrm{Hb}$ (Grigg, 1967). Grigg estudou o sangue integral em duas temperaturas $\left(-1,5^{\circ} \mathrm{C}\right.$ e $\left.4,5^{\circ} \mathrm{C}\right)$. Já que a variação da temperatura é uma em que se dá um número de transições de fase, um valor para $\Delta \mathrm{H}$ provavelmente não será calculado, especialmente sobre o sangue total. Ademais, Grigg (1967) mostrou que uma espécie ( $T$. berachii) tinha um efeito Root a $4,5^{\circ} \mathrm{C}$ porém não a- $1,5^{\circ} \mathrm{C}$. Entretanto, parece haver um efeito substancial da temperatura na afinidade de $\mathrm{O}_{2}-\mathrm{Hb}$ do sangue para estes peixes da Antártica.

A evidência mais forte para a reduzida sensibilidade térmica da afinidade de $\mathrm{O}_{2}-\mathrm{Hb}$ foi apresentada por diversos peixes de tuna e alguns de seus predatores. Por exemplo, a tuna de barbatana azul (Thunnus thynnus) nada por diversos meios térmicos e tem hemoglobinas que mostram pouco ou nenhuma sensibilidade à temperatura (Rossi-Fanelli e Antonini, 1960). Entretanto, esta adaptação da hemoglobina poderia ser mais um ajustamento à heterotermia interna do animal (Carey et al., 1966, 1969, 1971; e Carey, 1973) ao invés de uma adaptação ao meio térmico extremamente flutuante. $\mathrm{De}$ outro lado, podia-se argüir que qualquer adaptação é o resultado de mudanças moleculares e morfológicas e, por definição, estas mudanças não podem ser separadas. Se a adaptação é unicamente associada com as mudanças circulatórias internas, então, talvez é um exemplo inadequado para uma teoria generalizada de adaptação. Uma comunidade ainda mal conhecida é a da nêustonica ou animais que flutuam passivamente na superfície do mar. Um comprovante importante desta comunidade é o chamado "neustônico facultativo", esses animais que flutuam apenas à noite, descendo a $800-1000 \mathrm{~m}$ de profundidade durante 0 dia. Estas espécies experimentam grandes flutuações térmicas duas vezes por dia num curto período de natação através de uma termo clina $\left(\Delta T \geq 25^{\circ} \mathrm{C}\right)$. Infelizmente, nenhum estudo de temperatura foi feito sobre a afinidade de $\mathrm{O}_{2} \cdot \mathrm{Hb}$ dos peixes neustônicos facultativos, se não contam com metabolismo anaeróbio, (ver: Douglas et al., 1976), então os estudos futuros sobre estas espécies semelhantes poderiam aclarar esta questão.

Há alguns peixes poiquilotérmicos que vivem em meios térmicos anualmente flutuantes e têm hemoglobinas com sensibilidade reduzida à temperatura (e.g., Truta e C. clarkii) . $\mathrm{Em}$ tơdo caso, a redução na dependência à temperatura resulta da presença de um ou mais componentes raros de hemoglobinas são em geral acompanhadas por outros componentes que são relativamente insensiveis à temperatura. Essas hemoglobinas são em geral acompanhadas por outros componentes que são grandemente afetados pela temperatura. Já que a estratégia adaptativa parece ser semeIhante para a maioria destas espécies, uma discussão sobre os componentes I e IV da hemoglobina da truta poderia ser suficiente. A ligação de oxigênio da truta. Hbl é relativamente independente da temperatura com uma entalpia aparente, $\Delta \mathrm{H}$, entre $-3 \mathrm{e}-4 \mathrm{kcals} / \mathrm{mol}$ 
a $20^{\circ} \mathrm{C}$. Já que este valor é aproximadamente igual à temperatura da solução de oxigênio, a "entalpia intrínseca" é essencialmente zero. De outro lado, a HbIV de truta demonstra uma grande dependência à temperatura. $\mathrm{Em} \mathrm{pH}$ alcalino (i.e., onde os efeitos Root e Bohr são inoperantes), a entalpia é cerca de $-14 \mathrm{kcal} /$ mol. a qual é a mesma que a das hemoglobinas da maioria das outras espécies.

Foi proposto que a sensibilidade térmica reduzida da truta HbIV está possivelmente relacionada com as substituições de amino-ácido de $\beta 143$ (His $\rightarrow$ Phe), $\beta 143$ (His $\longrightarrow$ Ser) e talvez autras cadeias laterais de amino-ácido, (Barra, Bossa \& Bonaventura, 1973). Esta hipótese é reforçada pelos achados semelhantes em outras espécies de peixe (e.g., Powers \& Edmundson, 1972). Já que $\beta 146$ (His) foi mostrado ser responsável por uma grande quantidade do efeito Bohr (Kilmartin \& Wooton, 1970), não constitui surpresa que a $\mathrm{Hbl}$ da truta e de diversas outras hemoglobinas de peixes sejam também essencialmente independentes do $\mathrm{pH}$. Foi sugerido (Powers, 1972) que a independência ao $\mathrm{pH}$ destes componentes das hemoglobinas poderiam ser ecologicamente importantes durante a acidose transitória.

Wyman e coautores (1977) mostraram que o $\mathrm{Hbl}$ no estado $\mathrm{T}$ tem um $\Delta \mathrm{H}$ positivo quando reage com um ligante, enquanto o $\mathrm{Hbl}$ no estado $\mathrm{R}$ tem um $\Delta \mathrm{H}$ negativo. Portanto, não é surpreendente que na concentração média de oxigênio, a entalpia neta pudesse ser pequena para as hemoglobinas que sejam estrutural e funcionalmente semelhantes ao $\mathrm{Hbl}$ da truta.

Johansen \& Lenfant (1972) sugeriram que a dependência mais baixa à temperatura da afinidade de oxigênio do sangue pode ser uma adaptação a grandes flutuações de temperatura ambiental. Eles compararam Neoceratodus e Protopterus, os peixes pulmonados australianos e africanos respectivamente. A sensibilidade diminuída à temperatura da ligação do $\mathrm{O}_{2}$ pelo sangue de Neoceratodus é vista como uma vantagem para este peixe, que é um respirador aquático com exceção onde $\mathrm{o}_{2}$ é deficiente. O Protopterus, por outro lado, vive num meio relativamente estável termicamente e a ligaçẵo de oxigênio por seu sangue tem uma dependência normal à temperatura. Este é um argumento plausível, porém Amia o
Bowfin, vivem num ambiente com grande variação de temperatura, ainda assim tem o sangue com a mesma dependência à temperatura da ligação de oxigênio como a encontrada em Protopterus. Johansen \& Lenfant (1972) ten tam explicar esta aparente anomalia do Amia, apontando que a Amia é um respirador aquático em concentrações elevadas de oxigênio e um respirador aéreo obrigatório em baixo oxigênio dissolvido, porém esta característica é também compartilhada pelo Neoceratodus Johansen, (1970). Johansen \& Lenfant (1972) também sugerem que as arraias, habitantes das profundezas, vivem num meio termicamentes estável o que elas correlacionam com uma grande dependência à temperatura da ligação de $\mathrm{O}_{2}$ pelo sangue. Embora o meio ambiente da arraia possa ser termicamente mais estável do que o dos peixes interditais, tais como o Fundulus, o meio ambiente da arraia da água das regiōes temperadas tem flutuações anuais maiores à temperatura do que o das espécies tropicais discutidas acima (Anônimo, 1968) .

Como os peixes da Amazônia vivem em áreas onde a temperatura da água varia apenas alguns graus durante o ano, nossa descoberta de que suas hemoglobinas apresentam um efeito significativo à temperatura é consistente com o argumento evolucionário para os animais de "ambientes térmicos constantes" (e.g., Johansen \& Lenfant, 1972). Entretanto, as espécies de peixe de regiões temperadas em nosso estudo têm hemoglobinas com sensibilidade térmica semelhante à de suas contrapartes das regiões tropicais (Tabela I), o que não é consistente com a observação acima. Talvez, o melhor exemplo seja o vairäo eurialino Fundulus heteroclitus. O Fundulus tem uma limitada variação de residência de 36 metros (Lotrich, 1975) e vive num dos gradientes térmicos anuais mais excessivos do mundo (Anônimo, 1968; Powers e Powers, 1975) . Ademais, seu habitat intertidal é caracterizado por mudanças diárias significativas de temperaturas $\left(\Delta T=10^{\circ} \mathrm{C}\right.$ por dia). Sendo as hemoglobinas de Fundulus termicamente quase tão sensíveis quanto as dos peixes dos "ambientes térmicos constantes". (Tabela I), uma teoria generalizada com respeito à hemoglobina, habitat, e estabilidade térmica não parece ser justificável. 
Embora uma vantagem seletiva para uma hemoglobina termicamente insensível pudesse ser concebivelmente feita para algumas espécies raras, o desenvolvimento evolucionário generalizado ou a sensibilidade térmica reduzida da hemoglobina para peixes em meios térmicos flutuantes não é obrigatório. Se a evolução, na verdade, favorecer geralmente uma diminuição na sensibilidade à temperatura da afinidade do $\mathrm{O}_{2}-\mathrm{Hb}$ em peixes que experimentam grandes flutuações, então deve estar primariamente associada com a regulação do $\mathrm{pH}$ intracelular, com os níveis e tipos de fosfatos orgânicos, e com outros fenômenos de união de ligantes ao invés da seleção das hemoglobinas per se. Espera-se que os estudos comparativos futuros dos equilíbrios de oxigênio para as soluções de hemoglobina e sangue, sob condições uniformes de análise, estabelecerão a correlação, ou talvez nenhuma, entre a sensibilidade térmica e a variabilidade ambiental.

\section{AGRADECIMENTOS}

A porção experimental maior deste trabaIho foi completada a bordo do barco R/V "Alpha Helix" e foi financiado pela bolsa do NSF (PCM 75-06451) com o financiamento adicional da bolsa do NSF DEB 76-19877 (D.A.P.) e uma bolsa da National Geographic Society (D.A.P.). Os participantes noruegúeses (H.J. e V.E.H. Fyhn) receberam também financiamento do Norwegian Research Council For Science and the Humanities.

Somos gratos ao capitão e tripulantes do R/V "Alpha Helix" pela sua ajuda e paciência.

Particularmente, apreciamos a ajuda do Dr. Austen Riggs sem o que esta pesquisa não seria possível. Suas críticas a este manuscrito foram muito valiosas. Ademais, suas motivações altruísticas e direção tranqüila como cientista chefe a bordo de um navio muito lotado foi um exemplo a que nós todos podíamos aspirar.

\section{SUMMARY}

$\mathrm{V}$ e have measured the temperature dependence of the oxygen equilibria of blood and hemoglobin solutions from four neotropical and three temperate zone fishes. Significant differences exist in the heats of oxygenation of the bloods but not the hemoglobin solutions from different species. The differences in thermal sensitivity of the oxygenation of the bloods appear to depend on differences in intracellular $\mathrm{pH}$, organic phosphates and other ligand-linked variables and not the intrinsic enthalpies of the oxygenation of the hemoglobins themselves.

\section{BIBLIOGRAFIA}

ANDERSEN, M. E.; Olson, J. S. \& GibSON, Q. H.

1973 - Studies on ligand binding to hemoglobins from teleosts and el asmobranchs. J. Biol. Chem., 248 : 331-341.

ANONYMOUS

1968 - Surface water temperature and sal inlty: Atlantic Coast; North and South America. U. S. Dept. of Comm. Coast and Geodetic Survey, Washington, D.C., U.S.A.

ANTONINI, E. \& BRUNORI, M.

1971 - Hemoglobin and myoglobin in their Reactions with ligands. North Holland Publishing Co., $436 \mathrm{p}$.

BARCROFT, J. \& KING, W. O. R.

1909 - The effect of tempeature on the dissociation cuve of blood. J. Physiol. Lond., 39 : 374-384.

Barra, D.; Bossa, F.; Bonaventura \& Brunori, M.

1973 - Hemoglobin components from trout (Salmo Irideus) : determination of the carboxyl and amino terminal sequences and their functiona| implications. FEBS Lett. $35:$ 151-154.

BENESCH, R. E. \& BENESCH, R.

1970 - The reaction between diphosphoglycerate and hemoglobín. Fed. Proc. 29 : 1101-1104.

Binotti, S.; Glovenco, B.; Giardina,

ANTONINI, E. BRUNORI, M. \& WYMAN, J.

1971 - Studies on functional properties of fish hemoglobins. II. The oxygen equilibrium of the isolated hemoglobin components of trout blood. Archs. Biochem. Biophys., $142: 274-280$.

Bonaventura, J.; Gillen, R. \& Riggs, A.

1974 - The hemoglobin of the Gross-opterygerian fish, Latimeria chalumnae (Smith) - Subunit structure and oxygen equilibrium. Archs. Biochem. Biophys., 163 : 728-734.

CAMERon, J. N.

1970 - The influence of environmental variables on the haematology of the pinfish (Lagodon rhomboides) and striped mullet (Mugil cephalus). Comp. Biochem. Physiol., 32 : 175-192.

CAREY, F. G.

1973 - Fishes and Warm Bodies. Scl. Amer., 228 : $36-44$. 
Carey, F. G. \& Teal, J. H.

1966 - Heat conservation in tuna muscle. Proc. Nat. Acad. Sci., 56 : 1464-1469.

1969 - Regulation of body temperature by the bluefin tuna. Comp. Biochem. Physiol., 28 : 205-213.

Carey, F. G.; Teal, J. M.; Kanwisher, J. W. \&

LAWSON, K. D.

1971 - Temperature regulation in Tuna. Amer. Zool., 11: 137-145.

Denton, J. E. \& Yousef, M. F.

1975 - Seasonal changes in hematology of rainbow trout, Salmo gairdneri. Comp. Biochem. Physiol., 51A: 151-153.

De Wilde, M. A. \& Houston, A. H.

1967 - Haematological aspects of the thermoacclimatory process in the rainbow trout Salmo gairdneri. J. Fish Res. Bd. Can., 24 : 2267-2281.

Dill, D. B.; Edwards, H. T. \& FloRkin, M. A.

1932 - Properties of the blood of the skate, (Raja oscillata) Biol. Bull., 62 : 23-36.

DINTZIS, $\mathrm{H}$.

1952 - Ph. D. Thesis, Harvard Univ., Cambridge, Mass.

Douglas, E. L.; Friedl, W. A. \& Pickwell, G. V.

1976 - Fishes in oxygen-minimum, zones: blood oxygenation characteristics. Science, 191: 957-959.

FRY, F. E. J. \& HART, J. S.

1948 - The relation of temperature to oxygen consumption in the goldfish. Biol Bull., $94:$ 66-77.

Fyhn, U. E. H.; Fyhn, H. J.; Davis, B. J. POWers, D. A.;

FYNK, W. L. \& GARLICK, R. L.

1978 - Heterogeneidade de hemoglobinas nos peixes da Amazônia. Acta Amazonica 8(4) : Suplemento. (Este volume).

Gillen, R. G. \& Rigos, A.

1971 - The hemoglobins of a fresch-water teleost Cichlasoma cyanoguttatum : the effects of phosphorylated organic compounds upon oxygen equilibria. Comp. Biochem. Physiol., 38B : $585-595$.

GILLEN, R. \& RigGs, A.

1972 - Structure and function of the hemoglobins of the carp, Cyprinus carpio. J. Biol Chem., $247: 6039-6046$

Creaney, C. S. \& Powers, D. A.

1977a - Cellular regulation of an allosteric modifier of fish hemoglobin. Nature, 270: 73-74.

1977b- Allosteric modifiers of fish hemoglobins : in vitro and in vivo studies of the effect of ambient oxygen and $\mathrm{pH}$ on erythrocyte ATP concentrations. J. Exp. Zool., (in press).

Grigg, G. C

1967 - Some respiratory properties of the blood of four species of Antarctic fishes. Comp Biochem. Physiol., 23.: 139-148.
Hashimoto, K.; Yamaguchi, Y. \& MatsuUra, F.

1960 - Comparative studies on two hemoglobins of salmon. IV. Oxygen dissociation curve. Bull. Japan, Soc. Sci., Fisheries, 26: 827.

huzel, J. R. \& Proesser, C. L.

1974 - Molecular mechanisms of temperature compensation in poikilotherms. Physiol. Rev., 34 : 620-677.

Houston, A. H. \& CYR, D.

1974 - Thermoacclimatory variation in the hemoglobin system of goldfish (Carassius auratus) and rainbow trout (Salmo gairdneri). J. Exp. Biol., 61: 455-461.

JOHANSEN, K

1970 - Air breathing in fishes, In : Fish Physiology, (ed. HOAR, W. S. \& RANDALL, D. J.), Academic Press, $4: 532$.

JohANSEN, K. \& Lenfant, C.

1972 - A comparative approach to the adaptability of $\mathrm{O}_{2}-\mathrm{Hb}$ affinity in Oxygen Affinity of Hemoglobin and Red Cell Acid Base Status (ed ASTRUP, P. \& RORTH, M.) Academic Press, Munksgaard, Copenhagen, Denmark.

JOHANSEN, K. \& Weber, R. E.

1976 - On the adaptabiity of haemoglobin function to environmental conditions. In: Perspectives in Experimental Biology. (ed. DAVIES, P. SPENCER), New York, Pergamon Press, p. 212-234.

Johunsen, K.; Hanson, D. \& Lenfant, C.

1970 - Respiration in a primitive airbreather, Amia calva. Resp. Physiol., 9: 162-174.

Kil uartin, J. V. \& Wooton, J. F.

1970 - Inhibition of Bohr effect after removal of C-terminal histidines from haemoglobi $\beta$ chains. Nature, Lond., 228 : 766-767.

KROGH, A. \& LEITCH, I.

1919 - The respiratory function of blood in fishes. J. Physiol. Lond., 52: 288.

LENFANT, C. \& JohANSEN, K.

1968 - Respiration in the African lungfish, Protopterus aethiopicus. I. Respiratory properties of blood and normal patterns of breathing and gas exchange. J. Exp. Biol., 49: 437-452.

Lenfant, C.; Johansen K. \& Grgg, G. C.

1966 - Respiratory properties of blood and pattern of gas exchange in the lungfish, Neoceratopus forsteri. Resp. Physiol., 2: 1-21.

LOTRICH, V. A.

1975 - Summer home range and movements of Fundulus heteroclitus (Pisces: Cyprinodontidae) in a tidal creek. Ecology, 56:191-198

MIED, P. \& POWERS, D. A.

1977 - The hemoglobins of the killifish Fundulus heteroclitus: separation, characterization and a model for the subunit composition. J. Biol. Chem., (submitted). 
MUMm, D. P.; Atha, D. H. \& RigGs, A.

1978 - The hemoglobin of the common sting ray, Dasyatis sabina: structural and functional properties. Comp. Biochem. Physiol., (In press).

POWERS, D. A.

1972 - Hemoglobin adaptation for fast and slow water habitats in sympatric catostomid fishes. Science, 177 : 360-362.

1974 - Structure - function and molecular ecology of fish hemoglobins in Hemoglobins: comparative, olecular biology models for the study of disease. Ann. New York Acad. Sci., $241:$ 472-490.

Powers, D. A. \& Edmundson, A. B.

1972 - Multiple hemoglobins of catostomid fish I. Isolation and characterization of the isohaemoglobins from Catosomus clarkii. J. Biol. Chem., 247 : 6686-6693.

POWERS, D. A. \& POWERS, D.

1975 - Predicting gene frequencies in natural populations: a testable hypothesis in the isozymes, IV. Genetics and Evolution (ed. MARKERT, C.). New York, Academic Press, p. 63-84.

POWERS, D. A.; FYhN, H. J.; FYhN, U. E. H.;

Martin, J. P.; Garlick, R. L. \& WoOd, S. C.

- A comparative study of the oxygen equilibria of blood from $\mathrm{O}$ Genera of Amazonian fishes. Comp. Biochem. Physiol. (this issue).

Prosser, C. L.

1967 - Molecular mechanisms of temperature adaptation. Amer. Assoc. Adv. Sci. Washington, D.C.

1973 - Comparative animal physiology. 3rd. Edition W. B. Saunders Co., Philadelphia, Pa.

Riggs, A.

1970 - Properties of fish hemoglobins in Fish Physiology (ed. HOAR, W. S. \& RANDALL, D. J.). New York, Acadernic Press, 4 : 209-252.
Roor, R. W.

1931 - The resplratory function of the blood of marine fishes. Biol. Bull., 61.: 427456 .

Rossi-FANELLI, A. \& ANTONINI, E,

1960 - Oxygen equilibrium of hemoglobin from Thunnus thynnus Nature (Lond.) 188: 895-896.

Smellie, R. M. S. \& Pennock, J. F.

1976 - Biochemical adaptation to environmental change. Biochemical Society Simposium $n$ ? 41. Biochemical Society, London.

WEBER, R. E.

1975 - Respiratory properties of haemoglobin from eunicid polychaetes. J. Comp. Physiol., $99: 297-307$.

Weber, R. E.; Sullivan, B.; Bonaventura, J. \&

BONAVENTURA, C.

1976 - The hemoglobin system of the primitive fish Amis calva : isolation and functional characterization of the individual hemoglobin components. Biochim. Biophys. Acta, .434: 18-31.

WYMAN, J.

1964 - Linked functions and reciprocal effects in hemoglobin: a second look. Advances in Protein Chem., 19: 223-286.

Wyman, J.; Gill, S. J.; Noll, L.; Giardina, B.;

COLOSIMo, A. \& BRUNori, M.

1977 - The balance sheet of a hemoglobin: thermodynamics of $\mathrm{CO}$ binding by hemoglobin trout. In : J. Mol. Biol., 109: 195-205.

Yamaguchi, K.; Kochiyama, Y.;

Hashimoto, K. \& MatsuUra. F.

1962 - Studies on multiple hemoglobins of cel II. Oxygen dissociation curve and relative amounts of components. F. and S: Bull Japan Soc. Sci. Fisheries, 28: 192.

1963 - Studies on two hemoglobins of loach II. Oxygen dissociation curve. Bull. Jap. Soc. Sci. Fish., 29 : 180-188. 